\title{
Role of AmpD, OprF and penicillin-binding proteins in $\beta$-lactam resistance in clinical isolates of Pseudomonas aeruginosa
}

Correspondence

John Quale

jquale@downstate.edu

Received 21 October 2006

Accepted 22 February 2007

\author{
Simona Bratu, David Landman, Jyoti Gupta and John Quale
}

\author{
Division of Infectious Diseases, State University of New York Downstate Medical Center, \\ 450 Clarkson Avenue, Brooklyn, NY 11203, USA
}

\section{INTRODUCTION}

Pseudomonas aeruginosa is an opportunistic pathogen, affecting patients in intensive care areas and those with cystic fibrosis. Both intrinsic and acquired resistance mechanisms contribute to antibiotic resistance, and multidrug resistance is increasingly common. Derepression of the chromosomal AmpC $\beta$-lactamase or, less commonly, acquisition of a new $\beta$-lactamase, are commonly observed in $\beta$-lactam-resistant isolates. AmpR is a positive transcriptional regulator belonging to the LysR family of regulatory proteins (Bagge et al., 2002; Kong et al., 2005). Although laboratory-derived mutants of $P$. aeruginosa involving $a m p R$ have confirmed the regulatory role of this protein (Kong et al., 2005), mutations involving ampR are infrequently seen in clinical isolates overexpressing $a m p C$ (Campbell et al., 1997; Bagge et al., 2002; Juan et al., 2005; Quale et al., 2006). The gene ampD encodes a cytosolic $\mathrm{N}$-acetyl-anhydromuramil-L-alanine amidase that represses ampC expression; altering mutations in this gene have been noted in only a few clinical isolates with derepressed $a m p C$ activity (Langaee et al., 2000; Juan et al., 2005). In studies involving the strain PAO1, inactivation of $a m p D$ resulted in partial derepression of $a m p C$, and $a m p C$ remained inducible with cefoxitin (Juan

Abbreviations: DFAM, 6-carboxyfluorescein; DTAM, 6-carboxytetramethylrhodamine. et al., 2006). Two homologues of ampD have been described recently that are also involved in the regulation of $\operatorname{ampC}$ expression; loss of all three ampD-type genes led to very high-level stably derepressed expression of ampC (Juan et al., 2006).

It is also evident that other mechanisms may be involved in $\beta$-lactam resistance in isolates of $P$. aerguinosa. The association between reduced production of the porin OprD and carbapenem resistance has been verified. However, the role of OprF in antibiotic resistance remains controversial. OprF is the most common outer-membrane protein in $P$. aeruginosa and is most closely related to OmpA of Escherichia coli (Woodruff \& Hancock, 1989; Rawling et al., 1998; Brinkman et al., 2000). Although the poreforming structure of OprF has been questioned (Gotoh et al., 1989; Yoshihara \& Nakae, 1989), it appears that the $\mathrm{N}$-terminal half of the molecule can form channels (Nikaido et al., 1991; Bellido et al., 1992; Rawling et al., 1998; Brinkman et al., 2000). OprF-deficient isolates, obtained by either chemical or insertional mutagenesis, demonstrate poor growth in low-osmolarity media and altered morphology (Gotoh et al., 1989; Woodruff \& Hancock, 1989; Rawling et al., 1998). In one clinical isolate, loss of OprF following exposure to a fluoroquinolone was associated with a fourfold to tenfold increase in the MICs for ciprofloxacin, carbenicillin and ceftazidime but not imipenem (Piddock et al., 1992). However, diminished 
OprF had a minimal effect on antibiotic resistance in laboratory-derived strains and in another clinical isolate (Woodruff \& Hancock, 1988; Gotoh et al., 1989; Chamberland et al., 1990). Increased permeability due to the disruption of membrane integrity by the loss of OprF may explain the minimal effect that OprF deficiency has on antibiotic susceptibility (Hancock \& Woodruff, 1988; Woodruff \& Hancock, 1988).

Studies evaluating the role of penicillin-binding protein alterations on $\beta$-lactam resistance in $P$. aeruginosa have also been conflicting. The high-molecular-mass penicillinbinding proteins (1a, 1b, 2 and 3) carry essential functions corresponding to those found in E. coli (Curtis et al., 1979; Noguchi et al., 1979). The low-molecular-mass penicillinbinding proteins function as DD-carboxypeptidases and are not essential for cell viability (Curtis et al., 1979; Noguchi et al., 1979, 1985). The anti-pseudomonal cephalosporins appear to bind preferentially to PBP3 (Pucci et al., 1991), whilst carbapenems bind to PBP2 (Quinn et al., 1986). Two different serotypes of $P$. aeruginosa isolated from a patient with cystic fibrosis that developed resistance to piperacillin following exposure to that antibiotic had reduced affinity for most penicillin-binding proteins, and one strain appeared to have an altered PBP3 (Godfrey et al., 1981). However, no changes in penicillin-binding proteins were observed in three clinical isolates that developed resistance to imipenem (Quinn et al., 1986).

In this report, we examined the mechanisms contributing to $\beta$-lactam resistance in a large number of clinical isolates of $P$. aeruginosa. The roles of $\mathrm{AmpD}, \mathrm{OprF}$ and penicillinbinding proteins were examined in a large number of previously characterized isolates (Quale et al., 2006).

\section{METHODS}

Thirty-three clinical isolates of $P$. aeruginosa with varying degrees of $\beta$-lactam susceptibility were selected as described previously (Quale et al., 2006). Susceptibility testing and ribotyping were also performed as described previously (Quale et al., 2006). The isolates have been characterized for expression (measured by real-time RT-PCR) of $\operatorname{ampC}$ and $o p r D$, and have had the regulatory gene $\operatorname{ampR}$ amplified and sequenced (Quale et al., 2006). For all experiments, P. aeruginosa ATCC 27853 was used as the control isolate.

ampD and ampE studies. To search for possible mutations affecting important genes involved in $a m p C$ expression, $a m p D$ and $a m p E$ were characterized. Template DNA was obtained using a DNeasy kit (Qiagen). The following primer pairs were used for amplification and sequencing: $a m p D$ forward, 5 '-AACGACTGCAGCAATGTCAG- $3^{\prime}$, and ampD reverse, $5^{\prime}$-ATGCCGGGTCTTTTCTTTCT-3'; and ampE forward, 5'-GAAATCATGCGGACACGTT-3', and $a m p E$ reverse, 5'-AGGAAACATGACGTTCCTGG-3'. The PCR conditions were $94{ }^{\circ} \mathrm{C}$ for $15 \mathrm{~min}$, followed by 32 cycles of $94{ }^{\circ} \mathrm{C}$ for $60 \mathrm{~s}, 37^{\circ} \mathrm{C}$ for $60 \mathrm{~s}$ and $72{ }^{\circ} \mathrm{C}$ for $60 \mathrm{~s}$, with a final extension at $72{ }^{\circ} \mathrm{C}$ for $10 \mathrm{~min}$.

For isolates in which $a m p D$ could not be amplified, the following non-overlapping primers were employed: ampD2 forward, $5^{\prime}$ ACGTCATGTTTCCTCCTTGG-3', and ampD2 reverse, 5'-ATTAGAGTGGGGGCTCGTTC- $3^{\prime}$. To evaluate further the $a m p D$ operon in isolates with non-amplifiable $a m p D$, primers derived from the adjacent ampE gene (ampEfor 5'-GAGACTGTAGACCACCACCAGAAGGT-3') and PA4523 (PA4523rev 5'-ACAGCCTGTACCAGAAGATCGAACAG-3') were employed. Because of the possibility of a large insertion sequence (Bagge et al., 2002), PCR was performed using Accutaq DNA polymerase (Sigma).

Amplified products underwent bidirectional sequencing using an automated fluorescent dye-terminator sequencing system (Applied Biosystems). Sequences were compared with those of the PAO1 strain of $P$. aeruginosa using the BLAST program from the National Center for Biotechnology Information.

oprF expression studies. Expression of oprF was determined using real-time RT-PCR. An overnight culture was diluted $1: 100$ in Mueller-Hinton broth and grown to late exponential phase. RNA was isolated and treated with DNase using an RNeasy kit (Qiagen). A total of $2 \mu \mathrm{l} \mathrm{RNA}$ adjusted to a concentration of $25 \mu \mathrm{g} \mathrm{ml} \mathrm{g}^{-1}$ was used for all RT-PCR experiments; the housekeeping gene $r p o D$ was used as the normalizing gene (Quale et al., 2006). Primers for oprF expression were $o p r F$ forward (5'-CTTCGACAAGTCCAAGGTCA-3') and $o p r F$ reverse (5'-AAGTGGACGGGTACTGCTTC-3') and the probe for detection was $5^{\prime}$-DFAM-CGCTGACATCAAGAACCTGGCTG-DTAM- $3^{\prime}$. Primer and probe concentrations were adjusted to provide amplification efficiencies of $\sim 90-110 \%$ for all experiments. Real-time RT-PCR experiments were performed using the Brilliant QRT-PCR master mix (Stratagene). Samples were run in triplicate; virtually all individual results were within $0.5 \mathrm{C}_{\mathrm{t}}$ (cycle threshold) units of the averaged triplicate value. Controls run without reverse transcriptase confirmed the absence of contaminating DNA in any of the samples. Carboxy-X-rhodamine was included as a reference dye. Normalized expression of oprF was calibrated against corresponding mRNA expression by $P$. aeruginosa ATCC 27853; results are given as the relative expression of the mRNA compared with $P$. aeruginosa ATCC 27853.

Penicillin-binding protein analysis. Penicillin-binding proteins were assessed using a chemiluminescence assay, as described previously (Quale et al., 2003). Briefly, isolates were grown to late exponential phase in brain heart infusion broth. Cells were harvested by centrifugation, sonicated and cellular debris was removed by centrifugation. Membranes were collected by ultracentrifugation $\left(100000 \mathrm{~g}\right.$ for $40 \mathrm{~min}$ at $4{ }^{\circ} \mathrm{C}$ ) and resuspended in $10 \mathrm{mM}$ phosphate buffer ( $\mathrm{pH}$ 7.0). Penicillin-binding proteins were bound with biotinylated ampicillin $\left(200 \mu \mathrm{g} \mathrm{ml}^{-1}\right)$ for $10 \mathrm{~min}$, and the reaction stopped by the addition of penicillin $\left(120 \mathrm{mg} \mathrm{ml}^{-1}\right)$ and $20 \%$ aqueous Sarkosyl. Following SDS-PAGE on a $12 \%$ resolving gel, the proteins were transferred to a nitrocellulose membrane using the Trans-Blot SD transfer cell (Bio-Rad). Following washes in PBS and skimmed milk, the membrane was incubated in a $1: 5000$ dilution of streptavidin-horseradish peroxidase solution. Penicillin-binding proteins were visualized using the Opti-4CN detection kit (Bio-Rad).

\section{RESULTS AND DISCUSSION}

A total of 33 clinical isolates was examined; susceptibility and ribotyping data are given in Table 1 . Ten isolates were non-susceptible to ceftazidime and had increased expression of $a m p C$, defined as a tenfold or greater increase over the control. One other isolate (UL517) was intermediate in its susceptibility to ceftazidime and did not have increased expression of $\operatorname{ampC}$. A total of 3 isolates were susceptible to ceftazidime with increased expression of ampC, and the remaining 19 isolates were susceptible to ceftazidime without increased ampC expression (Table 1). 
Table 1. Ribotyping, susceptibility data, $A m p D$, and $A m p E$ identification, and relative expression of ampC, oprD and oprF in 33 clinical isolates of $P$. aeruginosa

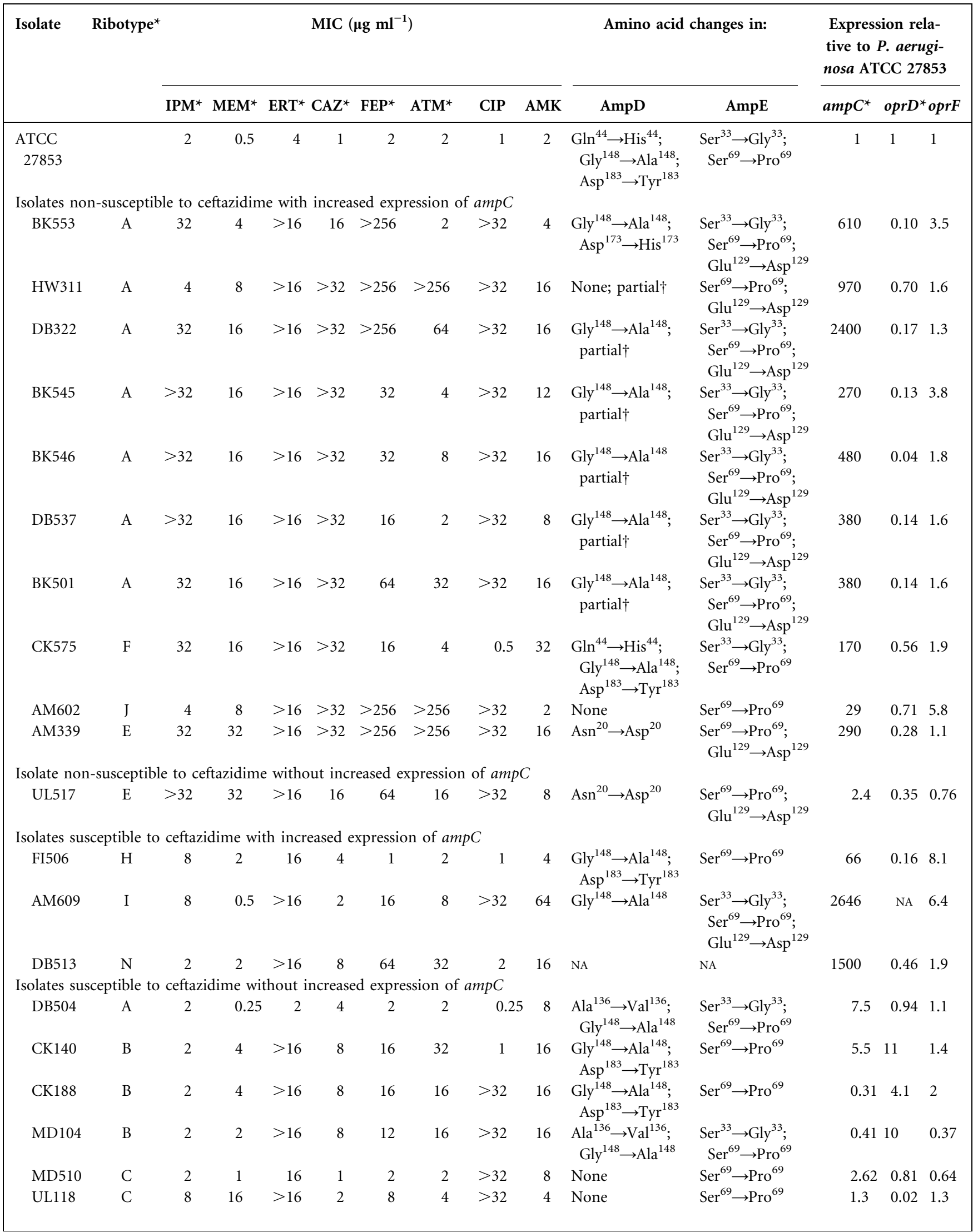


Table 1. cont.

\begin{tabular}{|c|c|c|c|c|c|c|c|c|c|c|c|c|c|c|}
\hline \multirow[t]{2}{*}{ Isolate } & \multirow[t]{2}{*}{ Ribotype $^{\star}$} & \multicolumn{8}{|c|}{$\operatorname{MIC}\left(\mu \mathrm{g} \mathrm{ml}^{-1}\right)$} & \multicolumn{2}{|c|}{ Amino acid changes in: } & \multicolumn{3}{|c|}{$\begin{array}{l}\text { Expression rela- } \\
\text { tive to } P \text {. aerugi- } \\
\text { nosa ATCC } 27853\end{array}$} \\
\hline & & IPM $^{*}$ & MEM $^{*}$ & ERT $^{\star}$ & $\mathrm{CAZ}^{*}$ & FEP $^{*}$ & $\operatorname{ATM}^{*}$ & CIP & AMK & AmpD & AmpE & $\operatorname{amp} C^{*}$ & $\operatorname{oprD} D^{\star}$ & ${ }^{*}$ oprF \\
\hline CK505 & C & 2 & 0.5 & 8 & 4 & 2 & 4 & 0.25 & 4 & None & $\operatorname{Ser}^{69} \rightarrow \operatorname{Pro}^{69}$ & 0.70 & 0.19 & 0.53 \\
\hline CK558 & $\mathrm{D}$ & 16 & 2 & $>16$ & 4 & 16 & 4 & 8 & 16 & None & $\begin{array}{l}\mathrm{Ser}^{69} \rightarrow \mathrm{Pro}^{69} \\
\mathrm{Leu}^{215} \rightarrow \mathrm{Phe}^{215}\end{array}$ & 2.0 & 0.38 & 0.55 \\
\hline CK550 & $\mathrm{D}$ & 8 & 2 & $>16$ & 4 & 16 & 4 & 4 & 16 & None & $\begin{array}{l}\mathrm{Ser}^{69} \rightarrow \mathrm{Pro}^{69} \\
\mathrm{Leu}^{215} \rightarrow \mathrm{Phe}^{215}\end{array}$ & 6.2 & 0.27 & 4.0 \\
\hline DB311 & $\mathrm{D}$ & 2 & 2 & $>16$ & 2 & 4 & 4 & 0.5 & 4 & None & $\begin{array}{l}\mathrm{Ser}^{69} \rightarrow \mathrm{Pro}^{69} \\
\mathrm{Leu}^{215} \rightarrow \mathrm{Phe}^{215}\end{array}$ & 1.4 & 1.8 & 3.2 \\
\hline BK555 & G & 32 & 4 & $>16$ & 2 & 8 & 4 & $>32$ & 8 & $\mathrm{Gly}^{148} \rightarrow \mathrm{Ala}^{148}$ & $\begin{array}{l}\text { Ser }^{33} \rightarrow \text { Gly }^{33} ; \\
\text { Ser }^{69} \rightarrow \text { Pro }^{69} ; \\
\text { Glu }^{129} \rightarrow \text { Asp }^{129}\end{array}$ & 2.4 & NA & 2.1 \\
\hline AM128 & $\mathrm{K}$ & 2 & 4 & 16 & 2 & 4 & 8 & 0.25 & 8 & $\begin{array}{l}\mathrm{Gln}^{44} \rightarrow \mathrm{His}^{44} ; \\
\mathrm{Gly}^{148} \rightarrow \mathrm{Ala}^{148}\end{array}$ & $\begin{array}{l}\text { Ser }^{33} \rightarrow \text { Gly }^{33} ; \\
\text { Ser }^{69} \rightarrow \text { Pro }^{69} ; \\
\text { Leu }^{215} \rightarrow \text { Phe }^{215}\end{array}$ & 3.6 & NA & 1.4 \\
\hline UL140 & $\mathrm{L}$ & 2 & 4 & $>16$ & 4 & 16 & 8 & $>32$ & 8 & None & $\begin{array}{l}\text { Ser }^{69} \rightarrow \text { Pro }^{69} \\
\text { Glu }^{129} \rightarrow \text { Asp }^{129}\end{array}$ & 5.1 & 2.4 & 0.89 \\
\hline MD119 & M & 2 & 4 & $>16$ & 4 & 8 & 8 & 0.25 & 4 & None & None & 2.7 & 1.2 & 0.23 \\
\hline MD519 & $\mathrm{O}$ & 2 & $\leqslant 0.12$ & 1 & 2 & 8 & 2 & 0.5 & 16 & $\mathrm{Gly}^{148} \rightarrow \mathrm{Ala}^{148}$ & $\mathrm{Ser}^{69} \rightarrow \mathrm{Pro}^{69}$ & 2.1 & NA & 1.1 \\
\hline MD504 & $\mathrm{P}$ & 2 & 0.25 & 2 & 2 & 2 & 4 & 0.25 & 8 & Gly $^{148} \rightarrow$ Ala $^{148}$ & $\begin{array}{l}\text { Ser }^{33} \rightarrow \text { Gly }^{33} ; \\
\text { Ser }^{69} \rightarrow \text { Pro }^{69} \\
\text { Glu }^{129} \rightarrow \text { Asp }^{129}\end{array}$ & 1.8 & NA & 0.89 \\
\hline MD521 & Q & 2 & 0.25 & 2 & 2 & 2 & 4 & 0.25 & 8 & $\mathrm{Gly}^{148} \rightarrow \mathrm{Ala}^{148}$ & $\begin{array}{l}\text { Ser }^{33} \rightarrow \text { Gly }^{33} ; \\
\text { Ser }^{69} \rightarrow \text { Pro }^{69} ; \\
\text { Glu }^{129} \rightarrow \text { Asp }^{129} \\
\text { Leu }^{190} \rightarrow \text { Phe }^{190}\end{array}$ & 3.8 & NA & 2.2 \\
\hline CK516 & $\mathrm{R}$ & 2 & $\leqslant 0.12$ & 2 & 2 & 1 & 2 & 0.12 & 4 & $\mathrm{Gly}^{148} \rightarrow \mathrm{Ala}^{148}$ & $\begin{array}{l}\text { Ser }^{33} \rightarrow \text { Gly }^{33} \\
\text { Ser }^{69} \rightarrow \text { Pro }^{69} \\
\text { Glu }^{129} \rightarrow \text { Asp }^{129} \\
\text { Leu }^{190} \rightarrow \text { Phe }^{190}\end{array}$ & 3.5 & NA & 1.4 \\
\hline CK503 & S & 1 & $\leqslant 0.12$ & 2 & 2 & 2 & 4 & 0.25 & 4 & $\begin{aligned} & \mathrm{Ala}^{136} \rightarrow \mathrm{Val}^{136} ; \\
& \mathrm{Gly}^{148} \rightarrow \mathrm{Ala}^{148}\end{aligned}$ & $\begin{array}{l}\mathrm{Ser}^{33} \rightarrow \mathrm{Gly}^{33} \\
\mathrm{Ser}^{69} \rightarrow \mathrm{Pro}^{69}\end{array}$ & 4.0 & 1.0 & 0.19 \\
\hline
\end{tabular}

AMK, Amikacin; ATM, aztreonam; CAZ, ceftazidime; CIP, ciprofloxacin; ERT, ertapenem; FEP, cefepime; IPM, imipenem; MEM, meropenem; NA, not amplifiable.

${ }^{\star}$ Data from Quale et al. (2006).

$\dagger$ Only the terminal $240 \mathrm{nt}$ of the gene could be amplified.

\section{$a m p D$ and $a m p E$ studies}

Most of the 33 clinical isolates had one or two amino acid substitutions involving ampD and ampE; these did not seem to affect expression of $a m p C$, as they were found in isolates with and without increased expression of ampC and were found in the control strain. Of the ten ceftazidime-resistant isolates with increased ampC expression, six (all from the same ribotype) had a deletion affecting the initial segment of $a m p D$ (Table 1). Attempts at amplification of the ampD operon using primers derived from the adjacent genes were also unsuccessful, suggesting genetic rearrangement involving this region. It is noteworthy that these six isolates did not have an inducible AmpC, consistent with constitutively derepressed mutants (Quale et al., 2006). A seventh isolate (BK553) in this group possessed a unique mutation causing an $\mathrm{Asp}^{173} \rightarrow \mathrm{His}^{173}$ change in AmpD; the significance of this mutation is unknown. One unique isolate (DB513) that was susceptible to ceftazidime, despite markedly increased expression of $a m p C$, had completely non-amplifiable $a m p D$ and ampE genes. Overall, of the 13 isolates with increased expression of $a m p C$, nearly half did not have changes in AmpD to explain the increased $\beta$-lactamase activity, results similar to those in another report involving clinical isolates (Juan et al., 2005). 


\section{oprF expression studies}

Most isolates had expression of oprF that approximated that of the control strain, $P$. aeruginosa ATCC 27853. The single isolate (UL517) that was non-susceptible to ceftazidime but without increased ampC expression had the lowest expression of oprF among the ceftazidimeresistant isolates (although it was comparable to many of the isolates in the ceftazidime-susceptible group). This isolate was intermediately susceptible to ceftazidime and aztreonam and resistant to cefepime, and did not overexpress efflux systems (Quale et al., 2006). Of the three isolates susceptible to ceftazidime with overexpression of ampC, two (AM609 and FI506) had markedly increased expression of $\operatorname{oprF}$ (6.4 and 8.1 times the control, respectively), suggesting that even increased $\beta$-lactamase activity could not overcome the increased permeability. The third isolate (DB513) had a markedly increased ampC expression but only marginally increased oprF expression; this isolate was resistant to cefepime and aztreonam, and the MIC for ceftazidime was at the breakpoint for susceptibility. It appears that, for selected isolates, the balance between $a m p C$ and $o p r F$ expression can affect the susceptibility to cephalosporins, although this interplay does not fully explain all of the susceptibility results we obtained.

For the 26 isolates in which there was both oprD and oprF expression data, there was a marked decrease in $\operatorname{opr} D$ in the isolates non-susceptible to imipenem, as expected $(0.21 \pm 0.15$ versus $2.7 \pm 3.6, P=0.03)$. These isolates also tended to have greater expression of $o p r F$, although this did not reach statistical significance $(2.6 \pm 2.1$ versus $1.5 \pm 1.5$, $P=0.15)$. There was no relationship with oprF expression and meropenem or ertapenem susceptibility.

\section{Penicillin-binding protein analysis}

The penicillin-binding proteins of 17 clinical isolates and $P$. aeruginosa 27853 were characterized (Fig. 1). All of the isolates appeared to have similar patterns regarding the essential high-molecular-mass proteins. Due to the close molecular masses of PBP4 and PBP5, there was poor resolution of these two non-essential proteins. Three isolates (including the control strain) had evidence of PBP6; the presence or absence of this protein did not appear to correlate with $\beta$-lactam susceptibility.

Resistance to cephalosporins in isolates of $P$. aeruginosa revolves around $\beta$-lactamase expression. Our findings suggest that expression of ampC generally correlates with cephalosporin resistance, although exceptions did occur. In some isolates, generally belonging to the same ribotype, major changes in $a m p D$ were found that could explain the increased expression of $a m p C$, although none had the previously reported insertion sequence or point mutation (Bagge et al., 2002). Many of our isolates did not have a readily identifiable reason for increased $a m p C$ expression, which has been noted in other clinical isolates (Juan et al., 2005).

Our findings also suggested that, for some isolates, there is a relationship between ampC and oprF expression and cephalosporin susceptibility. Whilst most of our isolates had oprF expression comparable to the control strain, occasional isolates had markedly increased expression of $o p r F$. For these isolates, increased expression of $a m p C$ did not result in cephalosporin resistance, suggesting that increased membrane permeability might offset the increase in $\beta$-lactamase activity. A modest inverse relationship between $\operatorname{oprF}$ and $\operatorname{oprD}$ was also noted in our clinical isolates. In one report, disruption of the regulatory gene $\operatorname{sig} X$ resulted in diminished oprF expression and increased production of a $47 \mathrm{kDa}$ outer-membrane protein (Brinkman et al., 1999); this may explain the slight decrease in the MIC of imipenem seen in one laboratory-derived OprF-deficient isolate (Woodruff \& Hancock, 1988).

In conclusion, our results suggest the following: (i) rearrangements in ampD are observed in some isolates overexpressing $\operatorname{amp} C$, although these isolates were generally clonally related, and for several unrelated isolates

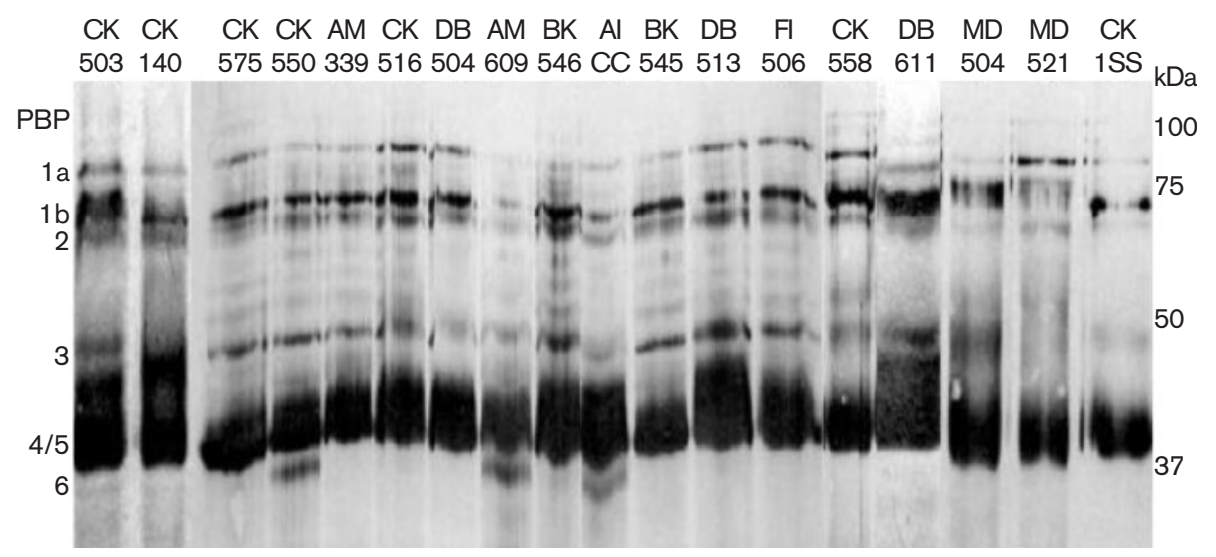

Fig. 1. Penicillin-binding protein patterns in 17 clinical isolates and in P. aeruginosa ATCC 27853 (lane 10). 
there was no apparent explanation for increased ampC expression; (ii) overexpression of oprF may offset increased ampC expression and isolates may retain susceptibility to cephalosporins; and (iii) penicillin-binding protein alterations did not appear to contribute to cephalosporin or carbapenem resistance.

\section{ACKNOWLEDGEMENTS}

This work was supported by a grant from Merck \& Co., Inc.

\section{REFERENCES}

Bagge, N., Ciofu, O., Hentzer, M., Campbell, J. I. A., Givskov, M. \& Høiby, N. (2002). Constitutive high expression of chromosomal $\beta$ lactamase in Pseudomonas aeruginosa caused by a new insertion sequence (IS1669) located in ampD. Antimicrob Agents Chemother 46, 3406-3411.

Bellido, F., Martin, N. L., Siehnel, R. J. \& Hancock, R. E. (1992). Reevaluation, using intact cells, of the exclusion limit and role of porin OprF in Pseudomonas aeruginosa outer membrane permeability. J Bacteriol 174, 5196-5203.

Brinkman, F. S. L., Schoofs, G., Hancock, R. E. W. \& De Mot, R. (1999). Influence of a putative ECF sigma factor on expression of the major outer membrane protein, OprF, in Pseudomonas aeruginosa and Pseudomonas fluorescens. J Bacteriol 181, 4746-4754.

Brinkman, F. S. L., Bains, M. \& Hancock, R. E. W. (2000). The amino terminus of Pseudomonas aeruginosa outer membrane protein OprF forms channels in lipid bilayer membranes: correlation with a threedimensional model. J Bacteriol 182, 5251-5259.

Campbell, J. I. A., Ciofu, O. \& Hoiby, N. (1997). Pseudomonas aeruginosa isolates from patients with cystic fibrosis have different $\beta$ lactamase expression phenotypes but are homogeneous in the ampCampR genetic region. Antimicrob Agents Chemother 41, 1380-1384.

Chamberland, S., Malouin, F., Rabin, H. R., Schollaardt, T., Parr, T. R., Jr \& Bryan, L. E. (1990). Persistence of Pseudomonas aeruginosa during ciprofloxacin therapy of a cystic fibrosis patient: transient resistance to quinolones and protein F-deficiency. J Antimicrob Chemother 25, 995-1010.

Curtis, N. A. C., Orr, D., Ross, G. W. \& Boulton, M. G. (1979). Competition of $\beta$-lactam antibiotics for the penicillin-binding proteins of Pseudomonas aeruginosa, Enterobacter cloacae, Klebsiella aerogenes, Proteus rettgeri, and Escherichia coli: comparison with antibacterial activity and effects upon bacterial morphology. Antimicrob Agents Chemother 16, 325-328.

Godfrey, A. J., Bryan, L. E. \& Rabin, H. R. (1981). $\beta$-Lactam-resistant Pseudomonas aeruginosa with modified penicillin-binding proteins emerging during cystic fibrosis treatment. Antimicrob Agents Chemother 19, 705-711.

Gotoh, N., Wakebe, H., Yoshihara, E., Nakae, T. \& Nishino, T. (1989). Role of protein $\mathrm{F}$ in maintaining structural integrity of the Pseudomonas aeruginosa outer membrane. J Bacteriol 171, 983-990.

Hancock, R. E. W. \& Woodruff, W. A. (1988). Roles of porin and $\beta$ lactamase in $\beta$-lactam resistance of Pseudomonas aeruginosa. Rev Infect Dis 10, 770-775.

Juan, C., Macia, M. D., Gutierrez, O., Vidal, C., Pérez, J. L. \& Oliver, A. (2005). Molecular mechanisms of $\beta$-lactam resistance mediated by
AmpC hyperproduction in Pseudomonas aeruginosa clinical strains. Antimicrob Agents Chemother 49, 4733-4738.

Juan, C., Moya, B., Perez, J. L. \& Oliver, A. (2006). Stepwise upregulation of the Pseudomonas aeruginosa chromosomal cephalosporinase conferring high-level $\beta$-lactam resistance involves three AmpD homologues. Antimicrob Agents Chemother 50, 1780-1787.

Kong, K.-F., Jayawardena, S. R., Indulkar, S. D., del Puerto, A., Koh, C.-L., Høiby, N. \& Mathee, K. (2005). Pseudomonas aeruginosa AmpR is a global transcriptional factor that regulates expression of AmpC and PoxB $\beta$-lactamases, proteases, quorum sensing, and other virulence factors. Antimicrob Agents Chemother 49, 4567-4575.

Langaee, T. Y., Gagnon, L. \& Huletsky, A. (2000). Inactivation of the ampD gene in Pseudomonas aeruginosa leads to moderate-basal-level and hyperinducible AmpC $\beta$-lactamase expression. Antimicrob Agents Chemother 44, 583-589.

Nikaido, H., Nikaido, K. \& Harayama, S. (1991). Identification and characterization of porins in Pseudomonas aeruginosa. J Biol Chem 266, 770-779.

Noguchi, H., Matsuhashi, M. \& Mitsuhashi, S. (1979). Comparative studies of penicillin-binding proteins in Pseudomonas aeruginosa and Escherichia coli. Eur J Biochem 100, 41-49.

Noguchi, H., Fukasawa, M., Komatsu, T., Mitsuhashi, S. \& Matsuhashi, M. (1985). Mutation in Pseudomonas aeruginosa causing simultaneous defects in penicillin-binding protein 5 and in enzyme activities of penicillin release and D-alanine carboxypeptidase. $J$ Bacteriol 162, 849-851.

Piddock, L. J. V., Hall, M. C., Bellido, F., Bains, M. \& Hancock, R. E. (1992). A pleiotropic, posttherapy, enoxacin-resistant mutant of Pseudomonas aeruginosa. Antimicrob Agents Chemother 36, 1057-1061.

Pucci, M. J., Boice-Sowek, J., Kessler, R. E. \& Dougherty, T. J. (1991). Comparison of cefepime, cefpirome, and cefaclidine binding affinities for penicillin-binding proteins in Escherichia coli K-12 and Pseudomonas aeruginosa SC8329. Antimicrob Agents Chemother 35, 2312-2317.

Quale, J., Bratu, S., Landman, D. \& Heddurshetti, R. (2003). Molecular epidemiology and mechanisms of carbapenem resistance in Acinetobacter baumannii endemic in New York City. Clin Infect Dis 37, 214-220.

Quale, J., Bratu, S., Gupta, J. \& Landman, D. (2006). Interplay of efflux system, $a m p C$, and $o p r D$ expression in carbapenem resistance of Pseudomonas aeruginosa clinical isolates. Antimicrob Agents Chemother 50, 1633-1641.

Quinn, J. P., Dudek, E. J., DiVincenzo, C. A., Lucks, D. A. \& Lerner, S. A. (1986). Emergence of resistance to imipenem during therapy for Pseudomonas aeruginosa infections. J Infect Dis 154, 289-294.

Rawling, E. G., Brinkman, F. S. L. \& Hancock, R. E. W. (1998). Roles of the carboxy-terminal half of Pseudomonas aeruginosa major outer membrane protein OprF in cell shape, growth in low-osmolarity medium, and peptidoglycan association. J Bacteriol 180, 3556-3562.

Woodruff, W. A. \& Hancock, R. E. W. (1988). Construction and characterization of Pseudomonas aeruginosa protein F-deficient mutants after in vitro and in vivo insertion mutagenesis of the cloned gene. J Bacteriol 170, 2592-2598.

Woodruff, W. A. \& Hancock, R. E. W. (1989). Pseudomonas aeruginosa outer membrane protein F: structural role and relationship to the Escherichia coli OmpA protein. J Bacteriol 171, 3304-3309.

Yoshihara, E. \& Nakae, T. (1989). Identification of porins in the outer membrane of Pseudomonas aeruginosa that form small diffusion pores. J Biol Chem 264, 6297-6301. 